\title{
How to Get a Degree-Anonymous Graph Using Minimum Number of Edge Rotations
}

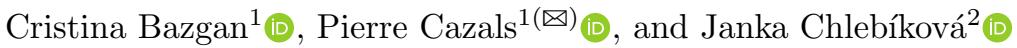 \\ 1 Université Paris-Dauphine, Université PSL, CNRS, LAMSADE, \\ 75016 Paris, France \\ \{cristina.bazgan, pierre.cazals\}@dauphine.eu \\ 2 School of Computing, University of Portsmouth, Portsmouth, UK \\ janka.chlebikova@port.ac.uk
}

\begin{abstract}
A graph is $k$-degree-anonymous if for each vertex there are at least $k-1$ other vertices of the same degree in the graph. Min Anonymous-EdGe-Rotation asks for a given graph $G$ and a positive integer $k$ to find a minimum number of edge rotations that transform $G$ into a $k$-degree-anonymous graph. In this paper, we establish sufficient conditions for an input graph and $k$ ensuring that a solution for the problem exists. We also prove that the Min Anonymous-Edge-Rotation problem is NP-hard even for $k=n / 3$, where $n$ is the order of a graph. On the positive side, we argue that under some constraints on the number of edges in a graph and $k$, Min Anonymous-Edge-Rotation is polynomial-time 2-approximable. Moreover, we show that the problem is solvable in polynomial time for any graph when $k=n$ and for trees when $k=\theta(n)$.
\end{abstract}

\section{Introduction}

Huge amounts of data has been aggregated on social networks in recent years. To assure the privacy of network's users is one of the key research task in this field. One possible study model was introduced by Liu and Terzi [15] who transferred the $k$-degree-anonymity concept from tabular data in databases [9] to graphs which are often used as a representation of networks. Therefore, a graph is called $k$-degree-anonymous if for each vertex there are at least $k-1$ other vertices with the same degree. The parameter $k$ represents the number of vertices that are mixed together and thus the increasing value of $k$ increases the level of anonymity. In [18], Wu et al. presented a survey of different anonymization models and some of their weaknesses.

In this paper we consider the $k$-degree-anonymous concept of Liu and Terzi [15]. Different graph operations of transforming a graph into a $k$-degreeanonymous one are considered in several papers where the operations maybe the following: delete vertex/edge, add vertex/edge, or add/delete of an edge (see the references later). One advantage in that approaches of vertex/edge deletion/adding is that a solution always exists since in the worst case scenario one

(C) Springer Nature Switzerland AG 2020

W. Wu and Z. Zhang (Eds.): COCOA 2020, LNCS 12577, pp. 242-256, 2020.

https://doi.org/10.1007/978-3-030-64843-5_17 
can consider the empty or the complete graph that is $k$-degree-anonymous for any $k$ (at most the number of vertices of the graph). However, the basic graph parameters as the number of vertices and edges could be modified.

In this paper we consider the version of transforming a graph into a $k$-degreeanonymous one using edge rotations which don't modify the number of vertices/edges, however a solution may not always exists, as we show later.

Vertex/edge modification versions associated to $k$-degree-anonymity have been relatively well studied. Hartung et al. [12,13] studied the edge adding modification as proposed by Liu and Terzi [15]. For this version Chester et al. [6] established a polynomial time algorithm for bipartite graphs

The variant of adding vertices instead of edges was studied by Chester et al. and in [5] they presented an approximation algorithm with an additive error. Bredereck et al. [2] investigated the parameterized complexity of several variants of vertex adding which differ in the way the inserted vertices can be adjacent to existing vertices. Concerning the vertex deletion variant, Bazgan et al. [1] showed the NP-hardness even on very restricted graph classes such as trees, split graphs, or trivially perfect graphs. Moreover, in [1] the vertex and edge deletion variants are proved intractable from the approximability and parameterized complexity point of view.

Several papers study the basic properties of edge rotations, including some bounds for the minimum number of edge rotations between two graphs $[3,4,8$, 14]. To the best of our knowledge the problem of transforming a graph to a $k$-degree anonymous graph using the edge rotations has not been fully explored. In some particular cases some research has been done, e.g. in [16] they study an edge rotation distance and various other metric between the degree sequences to find a "closest" regular graph.

Our Results. In this paper we study the various aspects of the MiN ANONYMOUsEDGE-Rotation problem. An input to the problem is an undirected graph $G=(V, E)$ with $n$ vertices and $m$ edges and an integer $k \leq n$. The goal is to find a shortest sequence of edge rotations that transforms $G$ into a $k$-degree-anonymous graph, if such a sequence exists. We first show that when $\frac{n}{2} \leq m \leq \frac{n(n-3)}{2}$ and $k \leq \frac{n}{4}$ a solution always exists. Moreover for trees a solution exists if and only if $\frac{2 m}{n}$ is an integer. We prove that Min Anonymous-EdGe-Rotation is NPhard even when $k=\frac{n}{3}$ and provide a polynomial-time 2-approximable algorithm under some constraints. Finally, we demonstrate that Min AnONYMOUS-EDGERotAtion is solvable in polynomial time for trees when $k=\theta(n)$ and for any graph when $k=n$.

Our paper is organized as follows. Some preliminaries about edge rotations and our formal definitions are given in Sect.2. The study of feasibility is established in Sect. 3. Section 4 presents the NP-hardness proof. In Sect. 5 we establish a lower bound that is used in Sect. 6 to present a polynomial-time 2-approximation algorithm and in Sect. 7 to demonstrate the polynomial time algorithm for trees. Moreover in Sect. 7 we consider the case $k=n$ in general graphs. Some conclusions are given at the end of the paper. The omitted proofs can be found in the full version of the paper. 


\section{Preliminaries}

In this paper we assume that all graphs are undirected, without loops and multiple edges, and not necessary connected graphs.

Let $G=(V, E)$ be a graph. For a vertex $v \in V$, let $\operatorname{deg}_{G}(v)$ be the degree of $v$ in $G$, and $\Delta_{G}$ be the maximum degree of $G$. A vertex $v$ with degree $\operatorname{deg}_{G}(v)=$ $|V|-1$ is called a universal vertex. The neighborhood of $v$ in $G$ is denoted by $\mathcal{N}_{G}(v)=\{u \in V: u v \in E\}$ and $\operatorname{Inc}_{G}(v)$ is the set of all edges incident to $v$, $\operatorname{Inc}_{G}(v)=\{e \in E: v \in e\}$. If the underlying graph $G$ is clear from the context, we omit the subscript $G$.

Definition 1. Given a graph $G=(V, E)$ of order $n$, the degree sequence $S_{G}$ of $G$ is the non-increasing sequence of its vertex degrees, $S_{G}=\left(\operatorname{deg}\left(v_{1}\right), \ldots, \operatorname{deg}\left(v_{n}\right)\right)$, $\operatorname{deg}\left(v_{1}\right) \geq \operatorname{deg}\left(v_{2}\right) \geq \cdots \geq \operatorname{deg}\left(v_{n}\right)$. A sequence $D$ of non-negative integers $D=\left(d_{1}, d_{2}, \ldots, d_{n}\right)$ is graphic if there exists a graph $G$ such that its degree sequence coincides with $D$.

As follows from Erdös-Gallai theorem (see e.g. [7]) the necessary and sufficient conditions for a non-increasing sequence $D=\left(d_{1}, d_{2}, \ldots, d_{n}\right)$ to be graphic are:

$$
\begin{aligned}
& \sum_{i=1}^{n} d_{i} \text { is even } \\
& \sum_{i=1}^{\ell} d_{i} \leq \ell(\ell-1)+\sum_{i=\ell+1}^{n} \min \left(d_{i}, \ell\right) \text { holds for any } 1 \leq \ell \leq n .
\end{aligned}
$$

Furthermore, it is an easy exercise to prove that a sequence of integers $D=$ $\left(d_{1}, d_{2}, \ldots, d_{n}\right)$ corresponds to a degree sequence of a tree on $n$ vertices if and only if each $d_{i} \geq 1$ and $\sum_{i=1}^{n} d_{i}=2(n-1)$.

Let $\mathbf{G}(n, m)$ be the set of all graphs with $n$ vertices and $m$ edges.

Definition 2. Let $G, G^{\prime} \in \boldsymbol{G}(n, m)$. We say that $G^{\prime}$ can be obtained from $G$ by an edge rotation $(u v, u w)$ if $V(G)=V\left(G^{\prime}\right)$ and there exist three distinct vertices $u, v$ and $w$ in $G$ such that $u v \in E(G), u w \notin E(G)$, and $E\left(G^{\prime}\right)=$ $(E(G) \backslash\{u v\}) \cup\{u w\}$.

Remark 1. Let $G$ be a graph. For the vertices $u, v, w$ in $G$ the edge rotation $(u v, u w)$ modifies $G$ into the graph $G^{\prime}$ such that $\operatorname{deg}_{G^{\prime}}(v)=\operatorname{deg}_{G}(v)-1$, $\operatorname{deg}_{G^{\prime}}(w)=\operatorname{deg}_{G}(w)+1$, and the degree of the other vertices is not changed. Let define a $(+1,-1)$-degree modification of the degree sequence $D=\left(d_{1}, \ldots, d_{n}\right)$ in such a way that $d_{i}:=d_{i}+1, d_{j}:=d_{j}-1$ for any two indices $i, j$ such that $i, j \in\{1, \ldots, n\}$. Note that each edge rotation corresponds to a $(+1,-1)$-degree modification, but not opposite.

Definition 3. A sequence of integers $D=\left(d_{1}, d_{2}, \ldots, d_{n}\right)$ is called $k$ anonymous where $k \in\{1, \ldots, n\}$, if for each element $d_{i}$ from $D$ there are at least $k-1$ other elements in $D$ with the same value. A graph $G$ is called $k$-degree-anonymous if its degree sequence is $k$-anonymous. The vertices of the same degree correspond to a degree class. 
In this paper we study the following anonymization problem:

Min Anonymous-Edge-Rotation

Input: $(G, k)$ where $G=(V, E)$ is an undirected graph and $k$ a positive integer, $k \in\{1, \ldots,|V|\}$.

Output: If there is a solution, find a sequence of a minimum number $\ell+1$ of graphs $G_{0}=G, G_{1}, G_{2}, \ldots, G_{\ell}$ such that $G_{i+1}$ can be obtained from $G_{i}$ by one edge rotation, and $G_{\ell}$ is $k$-degree-anonymous.

Note that a solution to the Min AnONymous-EDGE-Rotation problem may not exist for all instances, e.g. there is no solution if $G$ is a graph obtained from the complete graph $K_{n}, n \geq 6$, removing an edge together with $k=3$. Therefore, we are only interested in studying of feasible instances $(G, k)$ defined as an instance for which there exists a solution to Min AnONymous-EdGE-Rotation. Our initial study of sufficient conditions for feasibility is covered in Sect. 3.

Obviously, since all graphs are 1-degree-anonymous, we are only interested in cases where $k \geq 2$.

The decision version associated to Min Anonymous-Edge-Rotation is defined as follows for a feasible instance $(G, k)$ :

\section{ANONYMOUS-EDGE-ROTATION}

Input: $(G, k, r)$ where $G=(V, E)$ is an undirected graph, $k \in\{1, \ldots,|V|\}$, and $r$ be a positive integer.

Question: Is there a sequence of $\ell+1$ graphs $G_{0}=G, G_{1}, G_{2}, \ldots, G_{\ell}$ such that $\ell \leq r, G_{i+1}$ can be obtained from $G_{i}$ by one edge rotation, and $G_{\ell}$ is $k$-degree-anonymous?

We also consider the Min AnONYMous-EdGE-Rotation problem in restricted graph classes, e.g. trees. In that case we require that all graphs in the sequence $G_{0}, \ldots, G_{\ell}$ must be from the same graph class. Note that the problem can also be studied without this requirement, but the results may be different.

The following theorem highlights important properties of the edge rotations, the proof can be found e.g. in [4].

Theorem 1. For any two graphs $G, G^{\prime} \in \boldsymbol{G}(n, m)$ there exists a sequence of edge rotations transforming $G$ into $G^{\prime}$.

Corollary 1. For any two graphs $G, G^{\prime} \in \boldsymbol{G}(n, m)$, the edge distance between $G$ and $G^{\prime}$ is bounded by $2 m$.

\section{$3 \quad$ Feasibility}

As it was discussed in Sect. 2, the Min AnOnymous-Edge-Rotation problem does not have a solution for every input instance. It is not difficult to see that 
if a graph is 'almost' complete or 'almost' empty, then there are only restricted options on the number of different degree classes.

First we present some sufficient conditions for an instance to be feasible showing that if a graph is not 'almost' complete or an empty graph, then a solution of the problem exists for all $k \leq \frac{n}{4}$, where $n$ is the order of the graph.

Theorem 2. Let $G \in \boldsymbol{G}(n, m)$ such that $\frac{n}{2} \leq m \leq \frac{n(n-3)}{2}$ and $n \geq 4$. Then there exists a feasible solution for the Min ANONYMOUs-EDGE-RotATion problem, hence a $k$-degree-anonymous graph $G^{\prime} \in \boldsymbol{G}(n, m)$, for any $k \leq \frac{n}{4}$.

Proof. Let $m, n, k$ be fixed. Any graph $G \in \mathbf{G}(n, m)$ is a 1-degree-anonymous graph, hence we can suppose $k \geq 2$.

In the first part of the proof we describe a construction of a $k$-anonymous sequence $D=\left(d_{1}, d_{2}, \ldots, d_{n}\right)$ with property $\sum_{i=1}^{n} d_{i}=2 m$ for any $m, n, k$ satisfying the restriction of the theorem. In the second part we show that the sequence $D$ is graphic, hence that the sequence satisfies the conditions (1) and (2) from Sect. 2. As $\sum_{i=1}^{n} d_{i}=2 m$ is the condition for a constructed sequence, the property (1) trivially holds.

Now we construct three distinct $k$-anonymous sequences Type $1,2,3$ of integers based on the values of $k$ and $s \equiv 2 m \bmod n$. Denote by $d$ the average degree of the graph $G$ defined as $d=\left\lfloor\frac{2 m}{n}\right\rfloor$.

Type 1: $k \leq s \leq n-k$

Let $D_{1}=\left(d_{1}^{1}, d_{2}^{1}, \ldots, d_{s}^{1}, d_{1}^{2}, d_{2}^{2}, \ldots, d_{n-s}^{2}\right)$ be a sequence of positive integers where for all $i, 1 \leq i \leq s, d_{i}^{1}=d+1$ and for all $j, 1 \leq j \leq n-s$, $d_{j}^{2}=d$ (see Fig. 1). The sequence contains $n$ elements and it is easy to see that $\sum_{i=1}^{s}(d+1)+\sum_{j=1}^{n-s} d=2 m$.

Following the assumptions $s \geq k$ and $n-s \geq k$, therefore $D_{1}$ is a $k$-anonymous sequence.

Type $2: s<k$

Let $D_{2}=\left(d_{1}^{1}, d_{2}^{1}, \ldots, d_{s+k}^{1}, d_{1}^{2}, d_{2}^{2}, \ldots, d_{n-s-2 k}^{2}, d_{1}^{3}, d_{2}^{3}, \ldots, d_{k}^{3}\right)$ be a sequence of positive integers where for all $i, 1 \leq i \leq s+k, d_{i}^{1}=d+1$; for all $r, 1 \leq r \leq$ $n-s-2 k, d_{r}^{2}=d$; for all $j, 1 \leq j \leq k, d_{j}^{3}=d-1$ (see Fig. 1). The sequence contains $n$ elements and $\sum_{i=1}^{s+k}(d+1)+\sum_{j=1}^{k}(d-1)+\sum_{\ell=1}^{n-s-2 k} d=2 m$

Since $n \geq 4 k$ and $s<k, n-s-2 k \geq k, D_{2}$ is a $k$-anonymous sequence.

Type 3: $s>n-k$

Let $D_{3}=\left(d_{1}^{1}, d_{2}^{1}, \ldots, d_{k}^{1}, d_{1}^{2}, d_{2}^{2}, \ldots, d_{s-2 k}^{2}, d_{1}^{3}, d_{2}^{3}, \ldots, d_{k+n-s}^{3}\right)$ be a sequence of positive integers where for all $i, 1 \leq i \leq k, d_{i}^{1}=d+2$; for all $r, 1 \leq r \leq s-2 k$, $d_{r}^{2}=d+1$; for all $j, 1 \leq j \leq k+n-s, d_{j}^{3}=d$ (see Fig. 1). The sequence has $n$ elements and $\sum_{i=1}^{k}(d+2)+\sum_{j=1}^{k+n-s} d+\sum_{\ell=1}^{s-2 k}(d+1)=2 m$. 


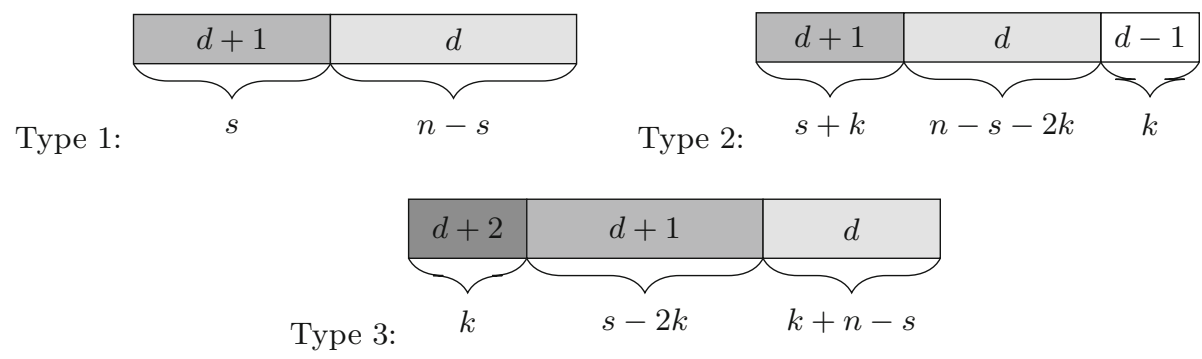

Fig. 1. The sequences of Type 1, 2, 3

Because $n>s$, the number $d$ appears more than $k$-times in $D_{3}$. Due to the assumptions $n \geq 4 k$ and $s>n-k$, also $s-2 k \geq k$. Hence $D_{3}$ is a $k$-anonymous sequence.

The proof that all three sequences are graphic can be found in the full version of the paper.

Now we extend the feasibility study to the case $k=n$ for which we get necessary and sufficient conditions.

Theorem 3. Let $G \in G(n, m)$ for some positive integers $n$ and $m$. Then $(G, n)$ is a feasible instance of Min AnOnymous-Edge-Rotation if and only if $\frac{2 m}{n}$ is an integer.

\section{NP-Hardness}

In this section we show that the decision version of MiN ANONYMOUs-EDGERotation, the problem Anonymous-Edge-Rotation, is NP-complete. The proof is based on a reduction from the restricted version of a cover set problem, Exact Cover By 3-Sets, which is known to be NP-complete [10].

Exact Cover By 3-Sets (X3C)

Input: A set $X$ of elements with $|X|=3 \mathrm{~m}$ and a collection $C$ of 3 -elements subsets of $X$ where each element appears in exactly 3 sets.

Question: Does $C$ contain an exact cover for $X$, i.e. a subcollection $C^{\prime} \subseteq C$ such that every element occurs in exactly one member set of $C^{\prime}$ ?

We define a polynomial-time reduction and then prove the NP-completeness of Anonymous-Edge-Rotation.

Reduction. Let $I=(X, C)$ be an instance of $X 3 C$ with $|X|=|C|=3 m$ and $m$ even. We describe the construction $\sigma$ transforming an instance $I$ into the graph $G:=\sigma(I)$ where $G=(V, E)$ is defined as follows:

- For each element $x \in X$, we add a vertex $v_{x}$ to the set $V_{\text {elem }} \subset V$ and a vertex $u_{x}$ to the set $V_{h u b} \subset V$. 
- For each 3 -element set $\{x, y, z\}$ of the collection $C$, we add 4 vertices $c_{x y z}^{1}$, $c_{x y z}^{2}, c_{x y z}^{3}$ and $c_{x y z}^{4}$ to the set $V_{\text {set }} \subset V$.

- For each $i \in\{1, \ldots, 5 m\}$ we add a vertex $w_{i}$ to the set $V_{\text {reg }} \subset V$ and for each $j \in\{1, \ldots, 10 m\}$ we add a vertex $t_{j}$ to $V_{\text {single }} \subset V$.

Let $V=V_{\text {elem }} \cup V_{\text {hub }} \cup V_{\text {set }} \cup V_{\text {reg }} \cup V_{\text {single. }}$. Obviously, $|V|=3 m+3 m+12 m+$ $15 m=33 m$. Now we define the set $E$ of the edges in $G$.

- For all $x, y \in X$, such that $x \neq y$, we add the edge $v_{x} u_{y}$ to $E_{X} \subset E$.

- For each 3-element set $\{x, y, z\}$ of the collection $C, \forall i \in\{1,2,3,4\}$, we add the edges $c_{x y z}^{i} u_{x}, c_{x y z}^{i} u_{y}$ and $c_{x y z}^{i} u_{z}$ to the set $E_{C} \subset E$.

- We add the set of edges $E^{\prime} \subset E$ to the vertex set $V_{\text {elem }}$ such that $\left(V_{\text {elem }}, E^{\prime}\right)$ is a 11-regular graph. Since the number of vertices in the set $\left|V_{\text {elem }}\right|=3 \mathrm{~m}$ is even ( $m$ is even) and $11<3 m$ such a regular graph exists [17]. Furthermore, such a graph can be constructed in polynomial time using Havel-Hakimi algorithm [11].

- We add the set of the edges $E^{\prime \prime} \subset E$ to the vertex set $V_{\text {reg }}$ such that $\left(V_{\text {reg }}, E^{\prime \prime}\right)$ is a $(3 m+11)$-regular graph. Since the number of vertices of $V_{r e g}$ is even and $3 m+11<5 m$

similarly to the previous case such a regular graph exists and can be constructed in polynomial time.

Finally, let $E=E_{X} \cup E_{C} \cup E^{\prime} \cup E^{\prime \prime}$.

Obviously, the graph $G=(V, E)$ has the following properties: (i) $10 \mathrm{~m}$ vertices of degree 0 (the vertices of the set $V_{\text {single }}$ ), (ii) $12 m$ vertices of degree 3 (the vertices of the set $V_{\text {set }}$ ), (iii) $8 m$ vertices of degree $3 m+11$ (the vertices of the set $V_{\text {reg }}$ and $V_{h u b}$ ), (iv) $3 m$ vertices of degree $3 m+10$ (the vertices of the set $\left.V_{\text {elem }}\right)$.

Theorem 4. Anonymous-Edge-Rotation is NP-complete even in case $k=$ $\frac{n}{3}$ where $n$ is the order of the graph $G$ for an input instance $(G, k, r)$.

Proof. Let $I=(X, C)$ be an instance of X3C with $|X|=|C|=3 m$ and consider the instance $I^{\prime}=(G, k, r)$ of Anonymous-Edge-Rotation where $G=\sigma(I)$, $k=11 m$ and $r=3 m$. We claim that $I$ is a yes-instance if and only if $I^{\prime}$ is a yes-instance.

Let $C^{\prime} \subseteq C$ be an exact cover for $X$ of size $m$. Now we define $3 m$ rotations which are independent from each other: for every 3-element set $\{x, y, z\} \in C^{\prime}$, we replace the edge $u_{x} c_{x y z}^{1}$ by the edge $u_{x} v_{x}$, and similarly $u_{y} c_{x y z}^{1}$ by $u_{y} v_{y}$ and $u_{z} c_{x y z}^{1}$ by $u_{z} v_{z}$. Since $C^{\prime}$ is of size $m$, we define exactly $3 m$ rotations. Let $G^{\prime}$ be the graph obtained from $G$ after applying all $3 m$ rotations. Since $C^{\prime}$ is an exact cover of size $m$ : (i) there are $m$ vertices of type $c_{x y z}^{1}$ that lost all 3 neighbours and become of degree 0 in $G^{\prime}$, (ii) all $3 m$ vertices of type $v_{x}$ are attached to a new neighbour, so they become of degree $3 m+11$ in $G^{\prime}$.

Then the graph $G^{\prime}$ has $10 m+m=11 m$ vertices of degree $0,12 m-m=11 m$ of degree 3 vertices, $8 m+3 m=11 m$ of degree $3 m+11$ vertices, hence we conclude that $G^{\prime}$ is the $11 m$-anonymous graph. 
Let $I^{\prime}$ be a yes-instance of Anonymous-EDGE-Rotation. Then there exists a sequence of $3 m$ rotations such that the graph $G^{\prime}=\left(V, E^{\prime}\right)$ obtained after applying the rotations to $G$ is a $11 m$-anonymous graph. Since $|V|=33 m$, there must be only three different degrees classes in $G^{\prime}$. Note that with one rotation, we can change the degree of two vertices, therefore the degree at most $6 \mathrm{~m}$ vertices can be changed by $3 m$ rotations. Since the graph $G$ has more than $6 m$ vertices of the degrees $3 m+11,3$, and 0 , all these degree classes must be in $G^{\prime}$. Furthermore, due to the number of vertices of $G$, these are the only degree classes in $G^{\prime}$. This means that in $G^{\prime}$ the number of vertices of degree $3 m+11$ must be increased by $3 m$, the number of vertices of degree 0 must be increased by $m$, the number of vertices of degree 3 must be decreased by $m$ and there are no vertices of degrees $3 m+10$ in $G^{\prime}$. A single rotation can increase or decrease the degree of a vertex by 1 therefore using $3 m$ rotations no vertex of degree $3 m+10$ in $G$ can have degree 0 in $G^{\prime}$ and similarly, no vertex of degree 3 in $G$ can have degree $3 m+11$ in $G^{\prime}$. Therefore the $3 m$ new vertices of degree $3 m+11$ in $G^{\prime}$ must have degree $3 m+10$ in $G$. This is only possible if the degree of each vertex $v_{x}$ from the set $V_{\text {elem }}$ is increased by 1 . Similarly, the $m$ new vertices of degree 0 in $G^{\prime}$ must have degree 3 in $G$, let $C_{G^{\prime}}$ be the set of such vertices. Obviously, $C_{G^{\prime}}$ must be a subset of $V_{\text {set }}$, in which the vertices have the form $c_{x y z}^{\ell}$ with $x, y, z \in X$, for any set $\{x, y, z\} \in C$, and $\ell \in\{1,2,3,4\}$.

To reach the requested degree configuration in $G^{\prime}$ with exactly $3 m$ edge rotations, in each rotation the degree of each vertex from $V_{\text {elem }}$ must be increased by 1 and the degree of each vertex from the set $C_{G^{\prime}}$ must be decrease by 1 . To achieve that, for each vertex $v_{x}$ from $V_{\text {elem }}$, the only possible rotation is to add the edge $u_{x} v_{x}$ where $u_{x} \in V_{h u b}$ and remove the edge $u_{x} c_{x y z}^{\ell}$ where $c_{x y z} \in C_{G^{\prime}}$. To fulfil the condition about the degree classes and the number of the rotations, the only way to achieve that is that $C^{\prime \prime}=\left\{\{x, y, z\} \mid c_{x y z}^{\ell} \in C_{G^{\prime}}\right\}$ is an exact cover of $X$.

\section{Lower Bound for a $k$-Degree-Anonymous Graph}

In this section we suppose that $(G, k)$ is a feasible instance. For any such instance we define a $k$-anonymous degree sequence $S_{\text {bound }}$ that can be computed in polynomial time if $k=\theta(n)$. We show that with the $(+1,-1)$-degree modifications (Remark 1) the graph $G$ can be transformed into a $k$-degree-anonymous graph $G^{\prime}$ with degree sequence $S_{\text {bound }}$ using at most double of edge rotations as in an optimal solution of Min AnONyMous-EdGe-Rotation for $(G, k)$.

Note that in general a $(+1,-1)$-degree modification doesn't correspond to an edge rotation, but as we show later in Sect. 7.1, it is true for trees.

Now in the following steps we show how to define the degree sequence $S_{\text {bound }}$.

\section{Step 1: Compute Every Available Target Sequence}

Let $S=\left(s_{1}, \ldots, s_{n}\right)$ be a non-increasing sequence of non-negative integers, $r \in$ $\{1, \ldots, n\}$. Any partition of $S$ into $r$ contiguous subsequences (i.e. if $S[a]$ and $S[b]$ are in one part, then all $S[i], a \leq i \leq b$ must be in the same part) is called a contiguous $r$-partition. The number of contiguous $r$-partitions of $S$ is $\left(\begin{array}{l}n-1 \\ r-1\end{array}\right)$, 
therefore bounded by $(n-1)^{r-1}$. Then the number of contiguous partitions of $S$ with at most $r$ parts can be bounded by $\sum_{i=0}^{r-1}(n-1)^{i} \leq 2 n^{r-1}$.

For each contiguous $\ell$-partition $p, 1 \leq \ell \leq r$, we use notation $p=\left[p_{1}, \ldots, p_{\ell}\right]$, where $p_{i}$ denotes the number of elements in part $i, 1 \leq i \leq \ell$. Note that at this stage important is the number of elements in each part, not which elements from $S$ are in it.

Let $G$ be a graph of order $n$ and $k$ an integer, $k \geq 2$. If $G$ is a $k$-degreeanonymous graph, then the vertices of $G$ can be partitioned into at most $c=\left\lfloor\frac{n}{k}\right\rfloor$ parts where the vertices in each part have the same degree. Let $P$ be the set of all such contiguous partitions with at most $c$ parts. As it follows from the initial discussion, the number of such partitions is bounded by $2 n^{c-1}$.

Now for each contiguous partition $p=\left[p_{1}, p_{2}, \ldots, p_{\ell}\right] \in P, \ell \in\{1, \ldots, c\}$, we compute all non-increasing sequences $\left(d_{1}, d_{2}, \ldots, d_{\ell}\right)$ of $\ell$ integers $d_{i}$ such that $0 \leq d_{i}<|V|$. Let $\hat{P}_{p}$ be the set of all feasible $k$-anonymous degree sequences for $p$, i.e.

$$
S=(\underbrace{d_{1}, \ldots, d_{1}}_{p_{1}-\text { times }}, \underbrace{d_{2}, \ldots, d_{2}}_{p_{2}-\text { times }}, \ldots, \underbrace{d_{\ell}, \ldots, d_{\ell}}_{p_{\ell}-\text { times }})=\left(d_{1}^{p_{1}}, d_{2}^{p_{2}}, \ldots, d_{\ell}^{p_{\ell}}\right) \in \hat{P}_{p}
$$

if and only if $\sum_{i=1}^{\ell} p_{i} d_{i}=2|E|, S$ is graphic and $k$-anonymous.

For each contiguous partition $p$ with $\ell$ parts, $1 \leq \ell \leq c$, there are at most $n$ possibilities for a degree on each position. The test whether the generated sequence is graphic and $k$-anonymous can be done in $O(n)$ operations. Since $|P|=O\left(n^{c-1}\right)$, there are at most $O\left(n^{c-1} \times n^{\ell} \times n\right) \leq O\left(n^{2 c}\right)$ operations to compute all feasible degree sequences of every partition, where $c=\left\lfloor\frac{n}{k}\right\rfloor$. Obviously, if $c$ is a constant, such number of operations is polynomial.

Step 2: Find the Best One

Now based on the previous analysis we can define the degree sequence $S_{\text {bound }}$ and prove some basic properties.

Definition 4. Let $G$ be a graph with the degree sequence $S_{G}$. Then define $S_{\text {bound }}$ for $G$ as a degree sequence for which the sum $\sum_{i=1}^{n}\left|S_{G}[i]-S[i]\right|$ achieves the minimum for all elements $S \in \hat{P}_{p}$ and $p \in P$.

Remark 2. Similarly to a $k$-anonymous sequence $S_{\text {bound }}$ defined in Definition 4 for a graph, we can define a $k$-anonymous sequence $S_{\text {Tbound }}$ for a tree. The only difference is that in the set $\hat{P}_{p}$, every feasible solution must have $d_{i} \geq 1$, which would be a subset of $\hat{P}_{p}$. Also for the testing, we don't need to check whether $S$ is graphic, the condition $\sum_{i=1}^{\ell} p_{i} d_{i}=2|E|$, is enough for the degree sequence of a tree.

The following lemmas describes the basic properties of sequences.

Lemma 1. Let $S$ be a n-sequence of non-negative integers and denote by $S^{\prime}$ the sequence $S$ sorted in non-increasing order. Let $S_{s}$ be another $n$-sequence of non-negative integers sorted in non-increasing order. Then 


$$
\left|\sum_{i=1}^{n}\right| S_{s}[i]-S^{\prime}[i]\left|\leq \sum_{i=1}^{n}\right| S_{s}[i]-S[i]
$$

Lemma 2. Let $(G, k)$ be a feasible instance for the Min AnOnymous-EdGeRotATION problem. Let OPT be an optimum solution that is a minimum set of rotations that transform $G$ to a $k$-degree-anonymous graph $G^{\prime}$. Then $\sum_{i=1}^{n} \mid S_{G}[i]-$ $S_{\text {bound }}[i]|\leq 2| O P T \mid$, where the degree sequence $S_{\text {bound }}$ is defined in Definition 4.

Proof. Let $S_{G^{\prime}}$ be the degree sequence of $G^{\prime}$ sorted in the same order as $S_{G}$ (i.e. for every $v \in V$, if $\operatorname{deg}_{G}(v)$ is in the position $i$ in $S_{G}$ then $\operatorname{deg}_{G^{\prime}}(v)$ is in the position $i$ in $S_{G^{\prime}}$. Let $S_{G^{\prime}}^{\prime}$ be the degree sequence $S_{G^{\prime}}$ sorted in nonincreasing order. As in the definition of $S_{\text {bound }}$ we considered all the options, there must exist $p \in P$ and $S \in \hat{P}_{p}$ such that $S=S_{G^{\prime}}^{\prime}$, and $\sum_{i=1}^{n}\left|S_{G}[i]-S_{\text {bound }}[i]\right| \leq$ $\sum_{i=1}^{n}\left|S_{G}[i]-S_{G^{\prime}}^{\prime}[i]\right|$.

Since the degree sequence $S_{G^{\prime}}^{\prime}$ is sorted in non-increasing order, then $\sum_{i=1}^{n}\left|S_{G}[i]-S_{G^{\prime}}^{\prime}[i]\right| \leq \sum_{i=1}^{n}\left|S_{G}[i]-S_{G^{\prime}}[i]\right|$ by Lemma 1 . One rotation from the graph $G_{j}$ to $G_{j+1}$ in the sequence of the graphs from $G$ to $G^{\prime}$ can only decrease the degree of a vertex by one and increase the degree of another one by one, hence $\sum_{i=1}^{n}\left|S_{G_{j}}[i]-S_{G^{\prime}}[i]\right| \leq \sum_{i=1}^{n}\left|S_{G_{j+1}}[i]-S_{G^{\prime}}[i]\right|+2$. This means by one rotation the value $\sum_{i=1}^{n} \mid S_{G}[i]-S_{G^{\prime}}[i]$ decreases by at most 2 . After $|O P T|$ rotations, the last graph $G_{j+1}$ in the sequence is $G^{\prime}$, therefore $\sum_{i=1}^{n}\left|S_{G}[i]-S_{G^{\prime}}[i]\right| \leq 2|O P T|$ and the lemma follows.

\section{Approximation}

In this section we show that under some constraints on the number of edges and $k$, there exists a polynomial time 2-approximation algorithm for the MIN Anonymous-Edge-Rotation problem for all feasible inputs $(G, k)$.

Remark 3. Let $S=\left(x_{1}, x_{2}, \ldots, x_{n}\right)$ be a non-increasing sequence of $n$ nonnegative integers. Denote by $R=x_{1}-x_{n}, A_{0}=\frac{x_{1}+x_{n}}{2}$, and let $A=\frac{\sum_{i=1}^{n} x_{i}}{n}$.

The standard deviation of $S$ is defined as $\sigma(S)=\sqrt{\frac{\sum\left(x_{i}-A\right)^{2}}{n}}$. It can be shown that

$$
\sum_{i=1}^{n}\left(x_{i}-A\right)^{2} \leq \sum_{i=1}^{n}\left(x_{i}-A_{0}\right)^{2} \leq \frac{n R^{2}}{4}
$$

hence $\sigma(S) \leq \frac{R}{2}$. 
The mean absolute derivation of $S$ is defined as $M A D[S]=\frac{1}{n} \sum_{i=1}^{n}\left|x_{i}-A\right|$. It is well known (e.g. applying Jensen's inequality) that $M A D[S] \leq \sigma(S)$.

Based on the correlation mentioned in Remark 3, we calculate an upper bound on the values in the degree sequence $S_{\text {bound }}$ in the following lemma.

Lemma 3. Let $(G, k)$ be an instance of the Min AnONymous-EdGERotation problem where $G$ is the graph with $n$ vertices and $m$ edges. Suppose that $\frac{n}{2} \leq m \leq \frac{n(n-3)}{2}, k \leq \frac{n}{4}$, and let the constant $c$ be defined as $c=\left\lfloor\frac{n}{k}\right\rfloor$, hence $k=\theta(n)$. Let $S_{\text {bound }}$ be the $k$-anonymous degree sequence associated with $G$ defined following Definition 4 . Then for every $i, S_{\text {bound }}[i] \leq$ $\min \left\{\left(1+\frac{n}{4 k}+\frac{n}{k \Delta}\right) \Delta, n-1\right\}, 1 \leq i \leq n$.

In the following two lemmas we prove that if a graph has 'sufficiently' many edges than edge rotations with the specific properties exist in a graph.

Lemma 4. Let $G=(V, E)$ be a graph with $|E|>\Delta^{2}$, let uv $\in E$. Then there exists an edge $a b \in E$ such that both vertices $a$ and $b$ are different from $u$ and $v$ and at most one of the following edges $\{a v, a u, b v, b u\}$ is in $E$.

Lemma 5. Let $G=(V, E)$ be a graph and suppose $|E|>\Delta^{2}$. Let $v^{+}, v^{-} \in$ $V$ such that $1 \leq d_{G}\left(v^{-}\right) \leq \Delta$ and $0 \leq d_{G}\left(v^{+}\right) \leq \Delta<|V|-1$. Then there exists a sequence of at most two edge rotations that transform $G$ to $G^{\prime}$ such that $d_{G^{\prime}}\left(v^{+}\right)=d_{G}\left(v^{+}\right)+1, d_{G^{\prime}}\left(v^{-}\right)=d_{G}\left(v^{-}\right)-1$ and degrees of other vertices in $G$ are not changed. These rotations can be found in $O\left(|E|^{2}\right)$ steps.

Theorem 5. The Min Anonymous-Edge-Rotation problem is polynomial time 2-approximable for all instances $(G, k), k \leq \frac{n}{4}$ where $k=\theta(n)$ and $G$ is the graph with $n$ vertices and $m$ edges, where $\max \left\{\frac{n}{2},\left(1+\frac{n}{4 k}+\frac{n}{k \Delta}\right)^{2} \Delta^{2}\right\} \leq m \leq$ $\frac{n(n-3)}{2}$, and the constant $c$ is defined as $c=\left\lfloor\frac{n}{k}\right\rfloor$.

Proof. Let $(G=(V, E), k)$ be an instance of Min Anonymous-EdgeRotation and $S_{G}$ be the degree sequence of $G$. Let the constant $c$ be defined as $c=\left\lfloor\frac{n}{k}\right\rfloor$. Due to our assumptions about the number of edges and $k$, all such instances are feasible as follows from Sect. 3 . First we compute a $k$-anonymous degree sequence $S_{\text {bound }}$ following Definition 4 in $O\left(n^{2 c}\right)$ steps. Due to the assumption $k=\theta(n)$ and consequently $c$ being a constant, such number of steps is polynomial. Furthermore, the condition on the number of edges ensures that we can always apply Lemma 5 and find suitable edge rotations.

If there exist two vertices $v^{+}, v^{-} \in V$ such that $0 \leq S_{G}\left[v^{+}\right]<S_{\text {bound }}\left[v^{+}\right] \leq$ $\left(1+\frac{n}{4 k}+\frac{n}{k \Delta}\right) \Delta<|V|-1$ and $S_{G}\left[v^{-}\right]>S_{\text {bound }}\left[v^{-}\right]$we apply Lemma 5 to transform $G$ to a graph $G_{1}$ with at most two rotations such that $d_{G_{1}}\left(v^{+}\right)=$ $d_{G}\left(v^{+}\right)+1$ and $d_{G_{1}}\left(v^{-}\right)=d_{G}\left(v^{-}\right)-1$.

We'll be executing the above transformations while there are two vertices $v^{+}$, $v^{-} \in V$ with the required properties. In each such transformation we decrease the degree of one vertex by 1 and increase the degree of another one by 1 with at most two rotations. Hence we transform $G$ to a final graph $G^{\prime}$ with degree 
sequence $S_{\text {bound }}$ by at most $\sum_{i=1}^{n}\left|S_{G}[i]-S_{\text {bound }}[i]\right|$ rotations. By Lemma 2 we know that $\sum_{i=1}^{n}\left|S_{G}[i]-S_{\text {bound }}[i]\right| \leq 2|O P T|$, hence we use at most 2 times the numbers of rotations of an optimal solution. In each transformation loop searching for the vertices $v^{+}$and $v^{-}$can be done in time $O(n)$ and searching for an edge $a b$ in time $O\left(\mathrm{~m}^{2}\right)$ (Lemma 4 ). Due to the modifications in each transformation loop, there can be at most $O\left(n^{2}\right)$ loops. Therefore the time complexity is bounded by $O\left(n^{2 c}+n^{2} \times m^{2} \times n\right)$. Since $c \geq 4, O\left(n^{2 c}+m^{2} \times n^{3}\right) \leq O\left(n^{2 c}\right)$.

Finally, since $S_{\text {bound }}$ is $k$-anonymous, $G^{\prime}$ is a $k$-degree-anonymous graph.

\section{Polynomial Cases}

As follows from Sect. 4, the Min Anonymous-Edge-Rotation problem is NPhard even for $k=\frac{n}{3}$, where $n$ is the order of an input graph. In this section we show that the problem can be solved in polynomial time on trees when $k=\theta(n)$ or in case of any graph when $k=n$.

\subsection{Trees}

For a tree $T=(V, E)$ rooted in a vertex $r$, for any $v \in V, v \neq r, \operatorname{child}(v)$ is a vertex that is a neighbor of $v$ not on the path from $r$ to $v$.

Lemma 6. Let $T=(V, E)$ be a tree and $v^{-}, v^{+}$vertices from $V$ such that $v^{-}$is not a leaf and $v^{+}$is not a universal vertex. Then using one rotation we can transform $T$ into a tree $T^{\prime}$ such that $d_{T^{\prime}}\left(v^{-}\right)=d_{T}\left(v^{-}\right)-1$ and $d_{T^{\prime}}\left(v^{+}\right)=$ $d_{T}\left(v^{+}\right)+1$.

Theorem 6. The Min AnOnymous-EDGE-Rotation problem is polynomialtime solvable for any instance $(T, k)$ where $T$ is a tree of the order $n, k \leq \frac{n}{4}$ and such that $c=\left\lfloor\frac{n}{k}\right\rfloor$ is a constant, hence $k=\theta(n)$.

Proof. Let $T$ be a tree and $S_{T}=\left(d_{1}, d_{2}, \ldots, d_{n}\right)$ its degree sequence sorted in non-increasing order. As it was mentioned in Sect. 2, for a degree sequence of a tree only the following conditions must hold $\sum_{i=1}^{n} d_{i}=2(n-1)$ and $d_{i} \geq 1$ for all $i, 1 \leq i \leq n$. Now based on $S_{T}$ define a $k$-anonymous sequence $S_{T b o u n d}$ as discussed in Sect. 5 .

Let $x$ and $y$ be integers such that $S_{T}[x]>S_{\text {Tbound }}[x]$ and $S_{T}[y]<S_{\text {Tbound }}[y]$. Since $S_{\text {Tbound }}$ correspond to a tree, $S_{\text {Tbound }}[x] \geq 1$ then $S_{T}[x]>1$ then $v_{x}$ is not a leave in $T$. Moreover since $S_{\text {Tbound }}[y] \leq n-1, v_{y}$ is not a universal vertex in $T$. 
By Lemma 6 there exists a tree $T_{1}$ such that $S_{T_{1}}[x]=S_{T}[x]-1$ and $S_{T_{1}}[y]=S_{T}[y]+1$. Repeat this operation until reaching a tree $T^{\prime}$ with the degree sequence $S_{\text {Tbound }}$. The cost of one operation is $O(n)$ and we repeat it $\sum_{i=1}^{n}\left|S_{T}[i]-S_{\text {Tbound }}[i]\right|$

2 times. Since $S_{\text {Tbound }}$ is $k$-anonymous, $T^{\prime}$ is a $k$-degreeanonymous tree. Since we use $\frac{\sum_{i=1}^{n}\left|S_{T}[i]-S_{\text {Tbound }}[i]\right|}{2} \leq|O P T|$ rotations (Lemma 2 ), the algorithm is optimal. The total cost of the algorithm is bounded by $O\left(n^{2 c}+n^{2}\right)=O\left(n^{2 c}\right)$, where $c=\left\lfloor\frac{n}{k}\right\rfloor$ is a constant.

\subsection{One Degree Class, $k=n$}

In this part we show that Min AnOnymous-EDGE-Rotation is polynomialtime solvable for instances where $k$ coincides with the number of vertices of the graph, that means all vertices must be in the same degree class.

Lemma 7. Let $G=(V, E)$ be a graph and $u, v \in V$. If $\mathcal{N}_{G}(u) \nsubseteq \mathcal{N}_{G}(v)$, then there is an edge rotation that leads to a graph $G^{\prime}$ such that $d_{G^{\prime}}(u)=d_{G}(u)-1$ and $d_{G^{\prime}}(v)=d_{G}(v)+1$.

Remark 4. Let $G=(V, E)$ be a graph, $\forall u, v \in V$, if $d_{G}(u)>d_{G}(v)$, then there is an edge rotation that leads to a graph $G^{\prime}$ such that $d_{G^{\prime}}(u)=d_{G}(u)-1$ and $d_{G^{\prime}}(v)=d_{G}(v)+1$.

Lemma 8. Let $(G, n)$ be an instance of Min AnOnymous-EdGeRotation where $G \in \boldsymbol{G}(n, m)$ for some positive integers $m, n$, and $\frac{2 m}{n}$ is an integer. Then the optimum value of Min AnONymous-EdGE-Rotation on $(G, n)$ is $\frac{\sum_{w \in V}\left|d_{G}(w)-2 m / n\right|}{2}$,

Theorem 7. The Min Anonymous-Edge-Rotation problem is polynomialtime solvable for instances $(G, k)$ when $k=n$, where $n$ is the order of the graph $G$.

Proof. In case $k=n$, we are looking for a $n$-degree-anonymous graph with only one degree class, hence for a regular graph. Due to Theorem 3, we can easily decide whether $(G, n)$ is a feasible instance of Min ANONYMOUs-EDGERotation: if for $G \in \mathbf{G}(n, m)$ the fraction $\frac{2 m}{n}$ is not an integer, $(G, n)$ is not a feasible input.

For a feasible input $(G, n)$, the result is based on Algorithm 1 and its correctness follows from Lemmas 7 and 8 .

Obviously, the algorithm runs in polynomial time. 


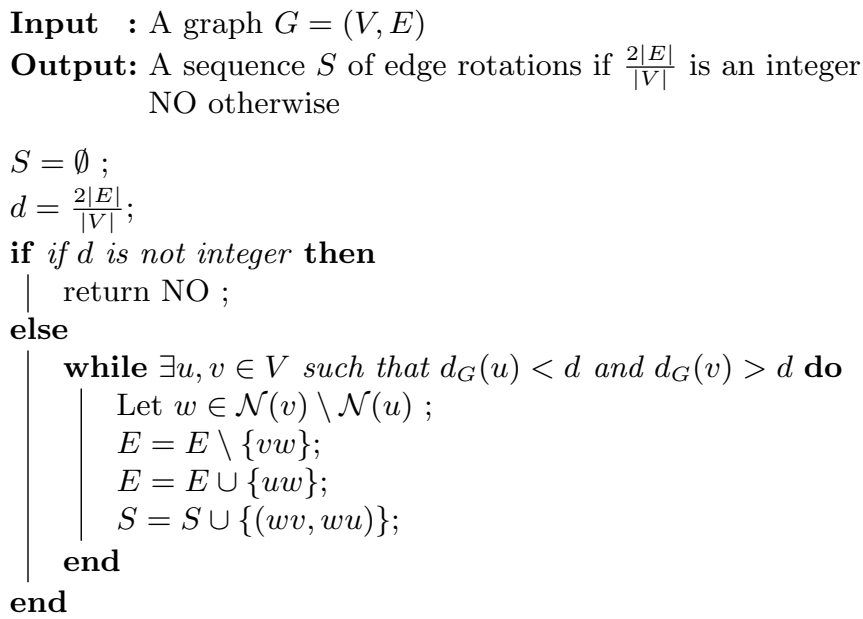

Algorithm 1: Algorithm for $k=|V|$

\section{Conclusion}

In this paper we initiate the study of the complexity of Min ANONYMOUS-EDGEROTATION problem in which the task is to transform a given graph to a $k$-degree anonymous graph using the minimum number of edge rotations. The problem doesn't have a solution for all graphs and all possible values of $k$, but our initial feasibility study already covers the majority of instances rotations. The problem doesn't have a solution for all graphs and all possible values of $k$, but our initial feasibility study already covers the majority of instances. The extensions of these results are still possible, mainly to find necessary and sufficient conditions for feasibility.

As we were able to prove NP-hardness in case where the number of vertices $k$ in each degree class is $\theta(n)$, further research could explore stronger hardness results or cases when $k$ is a constant. Our next research step includes relaxation of the condition on the number of the edges in the presented 2-approximation algorithm as well as extension of the graph classes in which the Min AnOnymousEDGE-Rotation problem can be solved in polynomial time.

\section{References}

1. Bazgan, C., Bredereck, R., Hartung, S., Nichterlein, A., Woeginger, G.J.: Finding large degree-anonymous subgraphs is hard. Theor. Comput. Sci. 622, 90-110 (2016)

2. Bredereck, Robert., Froese, Vincent., Hartung, Sepp., Nichterlein, André., Niedermeier, Rolf, Talmon, Nimrod: The complexity of degree anonymization by vertex addition. In: Gu, Qianping, Hell, Pavol, Yang, Boting (eds.) AAIM 2014. LNCS, vol. 8546, pp. 44-55. Springer, Cham (2014). https://doi.org/10.1007/978-3-31907956-1_5 
3. Chartrand, G., Gavlas, H., Johnson, H.H.M.A.: Rotation and jump distances between graphs. Discussiones Mathematicae Graph Theory 17, 285-300 (1997)

4. Chartrand, G., Saba, F., Zou, H.B.: Edge rotations and distance between graphs. Časopis pro pěstování matematiky 110(1), 87-91 (1985)

5. Chester, S., Kapron, B.M., Ramesh, G., Srivastava, G., Thomo, A., Venkatesh, S.: Why waldo befriended the dummy? k-anonymization of social networks with pseudo-nodes. Social Netw. Analys. Mining 3(3), 381-399 (2013)

6. Chester, S., Kapron, B.M., Srivastava, G., Venkatesh, S.: Complexity of social network anonymization. Social Netw. Analys. Mining 3(2), 151-166 (2013)

7. Erdos, P., Gallai, T.: Gráfok eloírt fokú pontokkal (graphs with points of prescribed degrees, in Hungarian). Mat. Lapok 11, 264-274 (1961)

8. Faudree, R.J., Schelp, R.H., Lesniak, L., Gyárfás, A., Lehel, J.: On the rotation distance of graphs. Discrete Math. 126(1-3), 121-135 (1994)

9. Fung, B., Wang, K., Chen, R., Yu, P.: Privacy-preserving data publishing: a survey of recent developments. ACM Comput. Surv. 42(4), 14:1-14:53 (2010)

10. Garey, M.R., Johnson, D.S.: Computers and Intractability, vol. 174. Freeman, San Francisco (1979)

11. Hakimi, S.L.: On realizability of a set of integers as degrees of the vertices of a linear graph. i. J. Soc. Ind. Appl. Math. 10(3), 496-506 (1962)

12. Hartung, S., Hoffmann, C., Nichterlein, A.: Improved upper and lower bound heuristics for degree anonymization in social networks. In: Gudmundsson, J., Katajainen, J. (eds.) SEA 2014. LNCS, vol. 8504, pp. 376-387. Springer, Cham (2014). https://doi.org/10.1007/978-3-319-07959-2_32

13. Hartung, S., Nichterlein, A., Niedermeier, R., Suchý, O.: A refined complexity analysis of degree anonymization in graphs. Inf. Comput. 243, 249-262 (2015)

14. Jarrett, E.B.: Edge rotation and edge slide distance graphs. Comput. Math. Appl. 34(11), 81-87 (1997)

15. Liu, K., Terzi, E.: Towards identity anonymization on graphs. In: Proceedings of the ACM SIGMOD International Conference on Management of Data, SIGMOD, pp. 93-106. ACM (2008)

16. Salas, J., Torra, V.: Graphic sequences, distances and k-degree anonymity. Discrete Appl. Math. 188, 25-31 (2015)

17. Tomescu, I.: Problems in combinatorics and graph theory. Wiley-Interscience Series Discrete Math. 212-213 (1961)

18. Wu, X., Ying, X., Liu, K., Chen, L.: A survey of privacy-preservation of graphs and social networks. In: Aggarwal, C., Wang, H. (eds.) Managing and Mining Graph Data. Advances in Database Systems, vol. 40, pp. 421-453. Springer, Heidelberg (2010). https://doi.org/10.1007/978-1-4419-6045-0_14 